\title{
Flavour Physics Technique at CDF and Do
}

\section{S. Donati ${ }^{\dagger}$}

University and INFN Pisa, Largo B. Pontecorvo, 3 - 56127 Pisa, Italy

E-mail: simone.donati@pi.infn.it

In this paper we review the detector elements and analysis techniques for heavy flavour physics analyses at CDF and D0. We describe the CDF and D0 detectors and triggers and we provide some example analyses, including the measurement of the $\mathrm{B}$ hadron masses, lifetimes, mixing and $\mathrm{CP}$ asymmetries.

Flavour Physics and CP Violation 2009

May 27 - June 1, 2009

Lake Placid, NY, USA

\footnotetext{
*Speaker.

${ }^{\dagger}$ for the CDF and D0 Collaborations
} 


\section{Introduction}

$\mathrm{B}$ hadrons are abundantly produced at the Tevatron Collider, where the measured $b$ production cross section $\left(\sigma\left(B^{+}\right)=2.78 \pm 0.24 \mu \mathrm{b}\right.$ for $B^{+}$mesons with $p_{T} \geq 6 \mathrm{GeV} / \mathrm{c}$ and $\left.|y| \leq 1,[1][2]\right)$ is three orders of magnitude larger than at $e^{+} e^{-}$machines running at the $\Upsilon(4 S)$, and the available energy allows the production of the heavier $B_{s}^{0}, B_{c}, \Lambda_{b}, \Sigma_{b}$ and $\Omega_{b}$ hadrons, as well as $B^{0}$ and $B^{+}$ mesons. The challenge is extracting the interesting $B$ signals from a level of background which is three orders of magnitude higher at production. This is achieved at CDF and D0 with dedicated detectors and triggers. With an integrated luminosity of about $6 \mathrm{fb}^{-1}$ (per experiment) now, we expect to have integrated $\sim 8 \mathrm{fb}^{-1}$ (per experiment) by the year 2010 .

\section{The CDF II detector and Trigger}

\subsection{Tracking Detectors}

The CDF II tracker is located within a $14.1 \mathrm{kG}$ solenoidal magnetic field and it is composed of silicon detectors and a drift chamber. There are three independent silicon detectors, SVXII, ISL and L00, for a total of eight silicon layer [3]. SVXII is made of double-sided ladders in a layout of five radial layers between 2.5 and $10.6 \mathrm{~cm}$ from the beamline. The ISL occupies the volume between SVXII and the drift chamber, with double-sided ladders at radii of 20 and 28 $\mathrm{cm}$. With a length of $1.9 \mathrm{~m}$, it provides silicon hits out to $|\eta|<2$. L00 is a single-sided layer of 48 ladders mounted directly on the beampipe, $1.5 \mathrm{~cm}$ from the beamline, which enhances the track impact parameter resolution. The Central Outer Chamber (COT, [4]) is located outside the silicon detectors and inside the time-of-flight detector scintillators. The active volume of the COT spans $310 \mathrm{~cm}$ in the beam direction, $43.4 \mathrm{~cm}$ and $132.3 \mathrm{~cm}$ in radius, and the entire azimuth. The $r-\phi$ view provides information for the $p_{T}$ measurement, the $r-z$ view for the $\eta$ measurement. The integrated CDF II tracker achieves a transverse momentum resolution of $\sigma\left(p_{T}\right) / p_{T}^{2}=0.15 \%$ $(\mathrm{GeV} / \mathrm{c})^{-1}$ and an impact parameter resolution of $\sigma(d)=35 \mu \mathrm{m} @ 2 \mathrm{GeV} / \mathrm{c}$.

\subsubsection{Particle Identification and Lepton Detectors}

CDF II uses two complementary techniques for particle identification, the $\mathrm{dE} / \mathrm{dx}$ measurement in the COT, and the time-of-flight measurement in a dedicated detector. The COT readout electronics allows to measure the pulse width, related to the amount of charge collected by the wire. The truncated mean $(80 \%)$ computed on the hits associated to a track provides a measurement of the specific ionisation $(\mathrm{dE} / \mathrm{dx})$ in the chamber. CDF achieves a $K / \pi$ separation for $p_{T}>2 \mathrm{GeV} / \mathrm{c}$ is $1.4 \sigma$. The Time-of-Flight detector (TOF, [5]) is installed between the drift chamber and the solenoid magnet. The detector is made of a total of 216 Bicron sintillator bars, each covering $1.7^{\circ}$ in $\phi$ and $|\eta|<1$. The time resolution on the single hit is $110 \mathrm{ps}$ and the $K / \pi$ separation is better than $2 \sigma$ for $p_{T}<1.5 \mathrm{GeV} / \mathrm{c}$. By combining the $\mathrm{dE} / \mathrm{dx}$ and the time-of-flight measurements, $\mathrm{CDF}$ achieves a $K / \pi$ separation better than $1.4 \sigma$ over the entire momentum range.

Segmented electromagnetic and hadronic calorimeters surround the tracking system [6]. The electron energy is measured by lead-scintillator sampling calorimeters. In the central region the calorimeters are arranged in a projective barrel geometry and measure electromagnetic energy with 
a resolution of $\left|\sigma\left(E_{T}\right) / E_{T}\right|^{2}=(13.5 \%)^{2} / E_{T}(\mathrm{GeV})+(2 \%)^{2}$. In the forward region the calorimeters are arranged in a projective end-plug geometry and measure the electromagnetic energy with a resolution of $\left|\sigma\left(E_{T}\right) / E_{T}\right|^{2}=(14.4 \%)^{2} / E_{T}(G e V)+(0.7 \%)^{2}$. The central muon detector ([7]) is located around the outside of the central hadron calorimeter. The pseudorapidity coverage is $|\eta|<0.6$. A second set of muon detectors is located behind an additional $60 \mathrm{~cm}$ of steel to reduce the fraction of fake muons. The coverage of the central muon system is extended to the region $0.6<|\eta|<1.0$ by four free-standing conical arches which hold drift chambers.

\subsubsection{Trigger and Data Acquisition}

CDF II uses a three-level system to reduce the $1.7 \mathrm{MHz}$ bunch crossing rate to $100 \mathrm{~Hz}$ written on tape. The Level 1 is a deadtimeless $7.6 \mathrm{MHz}$ synchronous pipeline with 42 cells, which allows $5.5 \mu$ s to form a trigger decision. The maximum sustainable Level 1 output rate is $\approx 30 \mathrm{kHz}$. The Level 2 is an asynchronous pipeline with an average latency of $20 \mu \mathrm{s}$. While the events accepted by Level 1 are being processed by Level 2 processors, they are also stored on one of the four Level 2 buffers, waiting for the Level 2 trigger decision. Each buffer is emptied when the Level 2 decision for the corresponding event has been asserted: if the event has been accepted, the buffer is read out, else it is simply cleared. If the Level 2 trigger decision takes too much time and the four buffers are all filled, the Level 1 accept is inhibited. This is a source of deadtime for the CDF II trigger. The Level 3 trigger is made of a CPU farm and has a maximum output rate of $100 \mathrm{~Hz}$.

The heart of the Level 1 trigger is the eXtremely Fast Tracker (XFT, [8] [9]), the trigger track processor that identifies high transverse momentum $\left(p_{T}>1.5 \mathrm{GeV} / \mathrm{c}\right)$ charged tracks in the COT. The XFT tracks are extrapolated to the calorimeter and to the muon chambers to generate electron and muon trigger candidates. The XFT efficiency is $\approx 90 \%$ per track, with a transverse momentum resolution better than $2 \%$ per $\mathrm{GeV} / \mathrm{c}$.

The Online Silicon Vertex Tracker (SVT, [10]) is part of the Level 2 trigger. It receives the list of XFT tracks and the digitised pulse heights on the axial layers of the silicon vertex detector. The SVT links the XFT tracks to the silicon hits and reconstructs tracks with offline-like quality. In particular the resolution on the impact parameter, which is a crucial parameter to select B events since they tipically show secondary vertices, is $35 \mu \mathrm{m}$ for $2 \mathrm{GeV} / \mathrm{c}$ tracks. SVT recontructs only tracks in the transverse plane to the beamline and only with $p_{T}>2.0 \mathrm{GeV} / \mathrm{c}$ with an efficiency of $85 \%$ per track. By providing a precision measurement of the impact parameter of the charged particle tracks, the SVT allows triggering on events cointaining long lived particles, like the B hadrons. Level 3 trigger is implemented on a CPU farm which allows to perform an almost offlinequality event reconstruction.

CDF II has basically three families of triggers for B physics: the dimuon trigger, the semileptonic trigger and the hadronic trigger. The dimuon trigger selects muon pairs with transverse momenum as low as $1.5 \mathrm{GeV} / \mathrm{c}$. It is mostly used to select $J / \psi \mathrm{s}$ and $\psi(2 S)$, to reconstruct the many decay modes of the $\mathrm{B}$ hadrons $\left(B^{0}, B^{+}, B_{s}^{0}, B_{c}\right.$, and $\left.\Lambda_{b}\right)$ containing a $J / \psi$ decaying to muon pairs, and to select $\Upsilon \rightarrow \mu^{+} \mu^{-}$decays, or muon pairs for the search of the rare $B \rightarrow \mu^{+} \mu^{-} X$ decays, or for $b \bar{b}$ correlation studies. The semileptonic trigger selects events with a lepton $(\mu$ or $e)$ with $p_{T}>4 \mathrm{GeV} / \mathrm{c}$ and an SVT track with $p_{T}>2 \mathrm{GeV} / \mathrm{c}$ and impact parameter above $120 \mu \mathrm{m}$. The hadronic trigger selects hadronic decay modes as $B_{(s)}^{0} \rightarrow h^{+} h^{\prime}$ and $B_{s}^{0} \rightarrow D_{s}^{-} \pi^{+}$by requiring track pairs with $p_{T}>2 \mathrm{GeV} / \mathrm{c}$ and impact parameter above $100 \mu \mathrm{m}$. 


\subsection{The D0 detector and trigger}

D0 is a general purpose detector [12] consisting of a central tracking system, uranium/liquidargon calorimeters and an iron toroid muon spectrometer. The D0 tracking system consists of a silicon microstrip tracker and a central fiber tracker, both located within a $2 \mathrm{~T}$ superconducting solenoidal magnet. The resolution for the distance of closest approach as provided by the tracking system is $\approx 50 \mu \mathrm{m}$ for tracks with transverse momentum $\approx 1 \mathrm{GeV} / \mathrm{c}$, improving to $15 \mu \mathrm{m}$ for tracks with transverse momentum above $10 \mathrm{GeV} / \mathrm{c}$. The central fiber tracker has eight thin coaxial barrels, each spporting two doublets of overlapping of scintillating fibers of $0.835 \mathrm{~mm}$ diameter, one doublet being parallel to the beam axis, and the other alternating by $\pm 3^{\circ}$. The silicon detector has four layers of double-sided detectors divided into six longitudinal sections interspersed with sixteen radial disks. Each layer has a side with strips parallel to the beam axis, two layers have a $\pm 2^{\circ}$ stereo side, and two layers have a $90^{\circ}$ side. The typical strip pitch is $50 \mu \mathrm{m}-80 \mu \mathrm{m}$. The accurate monitoring of the performance of the CDF and D0 silicon trackers does not show a significant degradation of the tracking performance with time. The muon system covers the region $|\eta|<2$ and is made of drift tubes. There are three layers of tubes with three or four planes/layer. Between layer 1 and layer 2 there is a toroid. The solenoid and toroid polarities of the muon detection system are reversed regularly, allowing a determination of the muon charge asymmetries induced by the detector. The calorimeter is made of Liquid Argon and uranium and has a depth of $\approx 20$ radiation lengths, ith a preshower detector made of scintillating strips.

D0 has two families of B triggers. The first family selects pairs of opposite charge muons (dimuon triggers), where each muon is required to have transverse momentum above $2.5 \mathrm{GeV} / \mathrm{c}$, and to have hits in at least two layers of the muon system, four layers in the central fiber tracker and three layers in the silicon trcker. The second family requires one muon (single muon triggers) with transverse momentun above $3 \mathrm{GeV} / \mathrm{c}$.

\section{Examples of world class analyses at CDF and D0}

CDF and D0 have reconstructed large samples of several B hadron leptonic and hadronic decay modes. $\mathrm{CDF}$ has performed $\mathrm{B}$ hadron mass measurements, which are fundamental physical observables used as an essential test of QCD, at sub-MeV level [18]. The B hadron decay modes used to perform the mass measurements are reconstructed in the $B^{+} \rightarrow J / \psi K^{+}, B^{0} \rightarrow J / \psi K^{* 0}$, $B_{s}^{0} \rightarrow J / \psi \phi, B^{0} \rightarrow J / \psi K_{s}^{0}$ and $\Lambda_{b}^{0} \rightarrow J / \psi \Lambda^{0}$ in the data sample collected by the $J / \psi$ trigger. For the mass measurement an accurate momentum scale calibration is essential. This has been performed using a sample of 1 million inclusive $J / \psi \rightarrow \mu^{+} \mu^{-}$decays to calibrate the energy loss in the detector material and the magnetic field, by removing any dependence on the transverse momentum of the $J / \psi$ mass.

CDF and D0 have recently reported the observation of the bottom, doubly-strange baryon $\Omega_{b}^{-}$through the decay chain $\Omega_{b}^{-} \rightarrow J / \psi \Omega^{-}$, where $J / \psi \rightarrow \mu^{+} \mu^{-}$and $\Omega^{-} \rightarrow \Lambda K^{-}$, and $\Lambda^{0} \rightarrow$ $p \pi^{-}$[13] [14]. D0 used $1.3 \mathrm{fb}^{-1}$ of data to observe a signal of $17.8 \pm 4.9$ (stat) \pm 0.8 (syst) events, with a significance $5.4 \sigma$, correspinding to a probability of $6.7 \times 10^{-8}$ of arising from a background fluctuation. CDF used $4.2 \mathrm{fb}^{-1}$ to observe a signal whose probability of arising from a background fluctuation is $4.0 \times 10^{-8}$, or 5.5 Gaussian standard deviations. The $\Omega_{b}^{-}$mass is 
measured to be $6054.4 \pm 6.8$ (stat.) \pm 0.9 (syst.) $\mathrm{MeV} / \mathrm{c}^{2}$. The lifetime of the $\Omega_{b}^{-}$baryon is measured to be $1.13_{-0.40}^{+0.53}$ (stat.) \pm 0.02 (syst.) ps. In addition, for the $\Xi_{b}^{-}$baryon we measure a mass of $5790.9 \pm 2.6$ (stat.) \pm 0.8 (syst.) $\mathrm{MeV} / \mathrm{c}^{2}$ and a lifetime of $1.56_{-0.25}^{+0.27}$ (stat.) \pm 0.02 (syst.) ps.

$\mathrm{CDF}$ and D0 perform also world class lifetime measurements of the $B^{0}, B^{+}, B_{s}^{0}$ mesons [15] [16] [17] . CDF has also performed the world best measurment of the $\Lambda_{b}^{0}$ baryon lifetime in the fully reconstructed and exclusive decay modes to $J / \psi[19]$. CDF uses the fully reconstructed decay mode $\Lambda_{b}^{0} \rightarrow J / \psi \Lambda^{0}$, with $J / \psi \rightarrow \mu^{+} \mu^{-}$and $\Lambda_{b}^{0} \rightarrow p \pi^{-}$. The measurement using fully reconstructed decays is competitive with the best previous measurements based on semileptonic decays. As compared to fully rconstructed decays, measurements using partially reconstructed semileptonic decays have additional uncertainties due to the missing energy of the unobserved neutrino and the modelling of background from other B baryons. With a similar technique and also as a crosscheck CDF measured the lifetime of the $B^{0}, B^{+}, B_{s}^{0}$ mesons in a topologically similar decay modes to $J / \psi$ states. CDF has also used the data collected by the hadronic trigger to perform $B^{0}, B^{+}$and $B_{s}^{0}$ lifetime measurements. The measurements in the decay modes $B^{+} \rightarrow \bar{D}^{0} \pi^{+}$and $B_{s}^{0} \rightarrow D_{s}^{-} \pi^{+}$. These measurements require an accurate estimate of the impact parameter trigger which introduces a bias on the B hadron lifetime distribution [20].

Particle identification is fundamental in several analyses, as the analysis of the $B_{(s)}^{0} \rightarrow h^{+} h^{-^{\prime}}$ decay modes which provided the first evidence of the $B_{s}^{0} \rightarrow K^{+} K^{-}, B_{s}^{0} \rightarrow K^{-} \pi^{+}$[21] and $\Lambda_{b}^{0} \rightarrow$ $p K^{-}$and $\Lambda_{b}^{0} \rightarrow p \pi^{-}$decay modes [22], and allowed to perform preliminary studies of CP violation in the $B_{s}^{0} \rightarrow K^{-} \pi^{+}$decay. The reconstructed $\pi \pi$-mass distribution shows a clean signal of $B_{(s)}^{0} \rightarrow h^{+} h^{\prime-}$ decays. In spite of a good mass resolution $\left(\approx 22 \mathrm{MeV} / \mathrm{c}^{2}\right)$, the various $B_{(s)}^{0} \rightarrow h^{+} h^{\prime}-$ mdoes overlap into an unresolved mass peak. The resolution in invariant mass and in particle identification is not sufficient for separating the individual decay modes on an event by event basis, therefore an unbinned maximum likelihood fit was performed, combining kinematic and particle identification information, to statistically determine both the contribution of each mode, and the relative contributions to the $\mathrm{CP}$ asimmetries. For the kinematic portion, three loosely correlated variables were used to summarise the information carried by all possible values of invariant mass of the B candidate, resulting from different mass assignments to the two outgoing particles. They are: (a) the mass $M_{\pi \pi}$ calculated with the charged pion mass assignment to both particles; (b) the signed momentum imbalance $\alpha=\left(1-p_{1} / p_{2}\right) q_{1}$, where $p_{1}\left(p_{2}\right)$ is the lower (higher) of the particle momenta, and $q_{1}$ is the sign of the charge of the particle momentum $p_{1}$ (Fig. 1); (c) the scalar sum of the particle momenta $p_{t o t}=p_{1}+p_{2}$. Particle identification information derives mostly from the $\mathrm{dE} / \mathrm{dx}$ measurement in the drift chamber which provides a $1.4 \sigma$ separation between pions and kaons in the momentum range of interest. A sample of $1.5 \mathrm{M} D^{*+} \rightarrow D^{0} \pi^{+} \rightarrow\left[K^{-} \pi^{+}\right] \pi^{+}$decays, where the $D^{0} D^{*+} \rightarrow D^{0} \pi^{+} \rightarrow\left[K^{-} \pi^{+}\right] \pi^{+}$decays, where the $D^{0}$ decay products are identified by the charge of the $D^{*+}$ pion, was used to calibrate the $\mathrm{dE} / \mathrm{dx}$ response over the tracking volume and over time (Fig. 1). The mass resolution function was parameterised using the detailed detector simulation. To take into account non-Gaussian tails due to the emission of photons in the final state, soft photon emission was included in the simulation using recent QED calculations [23]. The quality of the mass resolution was verified using about 500k $D^{0} \rightarrow K^{-} \pi^{+}$decays and improved agreement was found between data and the simulation including the final state radiation.

In addition to the improved measurement of branching fractions of the already known modes 
CDFII Monte Carlo

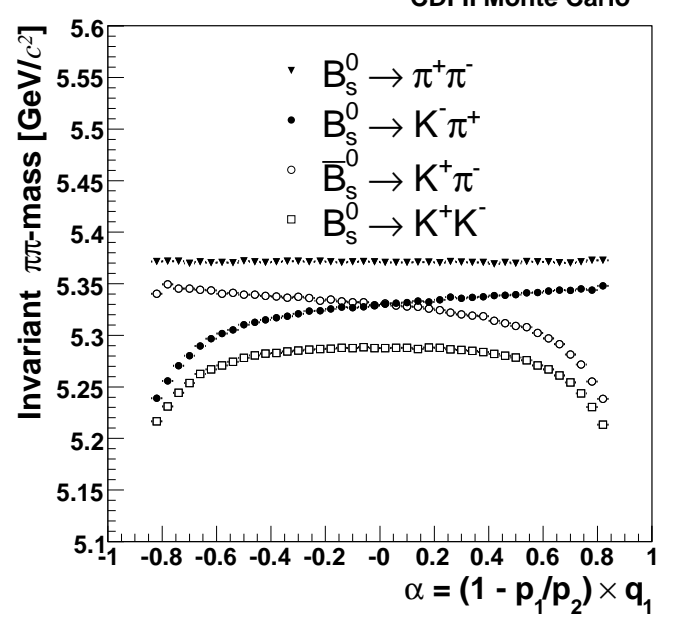

CDF Run II Preliminary

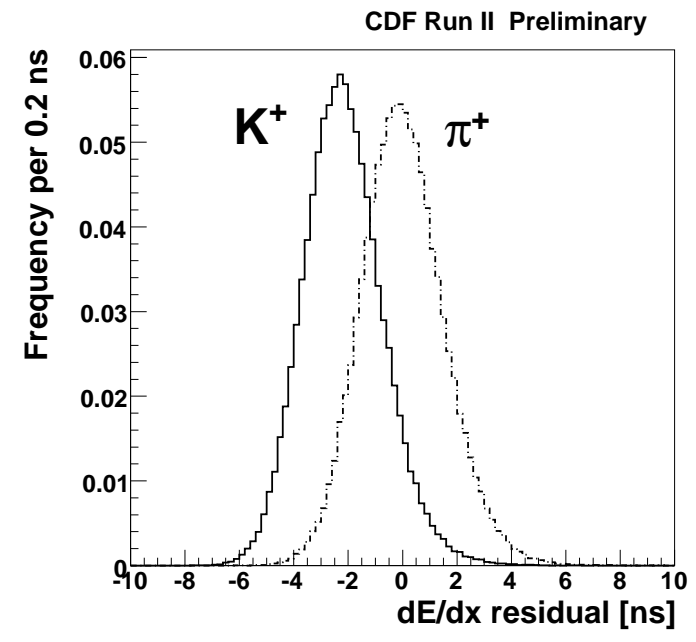

Figure 1: (Left plot:) $M_{\pi \pi}$ versus signed momentum imbalance $\alpha=\left(1-p_{1} / p_{2}\right) q_{1}$ for the $B_{s}^{0} \rightarrow h^{+} h^{\prime-}$ decays. Similar curves are for the $B_{d}^{0} \rightarrow h^{+} h^{\prime}$ and $\Lambda_{b}^{0} \rightarrow p h$ decays; (Rigt plot:) Tagged $D^{0} \rightarrow K^{-} \pi^{+}$decays from $D^{*+} \rightarrow D^{0} \pi^{+} \rightarrow\left[K^{-} \pi^{+}\right] \pi^{+}$: distribution of $\mathrm{dEdx}$ around the average pion response, for calibration samples of kaons and pions.

$\left(B^{0} \rightarrow \pi^{+} \pi^{-}, B^{0} \rightarrow K^{+} \pi^{-}\right.$and $\left.B_{s}^{0} \rightarrow K^{+} K^{-}\right)$and of the direct CP asymmetry $A_{C P}\left(B^{0} \rightarrow K^{+} \pi^{-}\right)$, the analysis observes three new rare modes for the first time $\left(B_{s}^{0} \rightarrow K^{-} \pi^{+}, \Lambda_{b}^{0} \rightarrow p \pi\right.$ and $\Lambda_{b}^{0} \rightarrow$ $p K$ ), with a significance respectively of $8.2 \sigma, 6.0 \sigma$ and $11.5 \sigma$, which includes both statistical and systematic undertainty. To convert the yields returned from the fit into relative branching fractions, we applied corrections for efficiencies of trigger and offline selection requirements for different decay modes.

The relative efficiency corrections between modes do not exceed $20 \%$. Most corrections were determined from the detailed detector simulation, with some exceptions which were measured using the data. The only correction needed for the direct CP asymmetries $A_{C P}\left(B^{0} \rightarrow K^{+} \pi^{-}\right)$and $A_{C P}\left(B_{s}^{0} \rightarrow K^{-} \pi^{+}\right)$was a $<0.6 \%$ shift due to the different probability for a $K^{+}$and $K^{-}$to interact with the tracker material. The measurement of this correction has been performed using a sample of $1 \mathrm{M}$ of prompt $D^{0} \rightarrow K^{-} \pi^{+}$decays reconstructed and selected using the same criteria as $B_{(s)}^{0} \rightarrow$ $h^{+} h^{\prime}-$ decays. Assuming the standard model expectation of $A_{C P}\left(D^{0} \rightarrow K^{-} \pi^{+}\right)=0$, the difference between the number of reconstructed $D^{0} \rightarrow K^{-} \pi^{+}$and $\vec{D}^{0} \rightarrow K^{+} \pi^{-}$provides a measurement of the detector induced asymmetry between $K^{+} \pi^{-}$and $K^{-} \pi^{+}$. The measured branching fraction of the newly observed mode $B R\left(B_{s}^{0} \rightarrow K^{-} \pi^{+}\right)=(5.0 \pm 0.75 \pm 1.0) \times 10^{-6}$ is in agreement with the latest theoretical predictions.

The analysis measures for the first time the direct CP asymmetry $A_{C P}\left(B_{s}^{0} \rightarrow K^{-} \pi^{+}\right)=0.39 \pm$ $0.15 \pm 0.08$, which favors a large $\mathrm{CP}$ violation in the $B_{s}^{0}$ decays. A robust test of the standard model can be performed by comparing the measurements of the direct $\mathrm{CP}$ asymmetry in the $B_{s}^{0} \rightarrow$ $K^{-} \pi^{+}$and $B^{0} \rightarrow K^{+} \pi^{-}$decays. The estimated expected value for $A_{C P}\left(B_{s}^{0} \rightarrow K^{-} \pi^{+}\right) \approx 0.37$ is in agreement with the CDF II measurement. 


\section{Flavor tagging}

Flavour tagging is crucial to study neutral $\mathrm{B}$ mixing and $\mathrm{CP}$ violation. The complicated hadron environment needs sophisticated techniques to achieve a good efficiency and purity of the tags [24]. There are two categories, the Opposite Side Taggers (OST) use the decay products of the opposte B in the event, the Same Side Taggers (SST) use the charge correlation of the fragmentation tracks produced on the same side of the reconstructed $\mathrm{B}$.

CDF uses the large samples of semileptonic B decays collected by the lepton+displaced track trigger to study the performance of the Opposite Side Lepton Taggers. The B component is enhanced by requiring $2 \mathrm{GeV} / \mathrm{c}^{2}<M_{l+t r k}<4 \mathrm{GeV} / \mathrm{c}^{2}$ and a positively displaced signed impact parameter of the track. The sign of the B flavor on the decay side is given by the trigger lepton associated to the opposite side B. The Soft Muon Tagger has an $\varepsilon D^{2}$ of $0.54 \%(1.48 \%)$ at CDF(D0), and the Soft electron Tagger has an $\varepsilon D^{2}$ of $0.29 \%(0.21 \%)$ at $\mathrm{CDF}(\mathrm{D} 0)$. The Jet Charge Tagger computes an average $b$ jet charge $Q_{j e t}=\frac{\sum q_{i} p_{T}^{-}}{\sum p_{T}^{i}}$, where $q_{i}$ and $p_{T}^{i}$ are respectively the charge and the transverse momenta of the tracks found within the $b$ jet. The Jet Charge Tagger has an $\varepsilon D^{2}$ of $0.23 \%(0.50 \%)$ at $\mathrm{CDF}(\mathrm{D} 0)$. CDF utilizes $\mathrm{dE} / \mathrm{dx}$ in the chamber and the TOF detector to identify kaons. This allows to exploit the correlation of the charge of the kaon with the B flavor in the decay chain $b \rightarrow c \rightarrow X K^{-}$. This tagger has $\varepsilon D^{2}$ of $0.23 \%$.

The Same Side Tagger is calibrated through the use of large Monte Carlo samples. This method makes heavy use of the particle identification techniques to identify the fragmentation kaon in the case of of $B_{s}^{0}$. The Monte Carlo calibration is performed by achieving good data-Monte Carlo agreement in large $B^{0}$ and $B^{+}$samples. CDF achieves an $\varepsilon D^{2}$ of $4.0 \%$.

The development of the flavour tagging techniques has been fundamental for the observation of the $B_{s}^{0}-\bar{B}_{s}^{0}$ oscillations performed by CDF [25] and D0 [26] using a time-dependent analysis. CDF has reconstructed a sample of 5600 fully reconstructed hadronic $B_{s}^{0}$ decays, 3100 partially reconstructed hadronic $B_{s}^{0}$ decays, and 61500 partially reconstructed semileptonic $B_{s}^{0}$ decays. CDF has measured the probability as a function of proper decay time that the $B_{s}^{0}$ decays with the same, or opposite, flavour as the flavour at production, and this techniques finds a signal for $B_{s}^{0}-\bar{B}_{s}^{0}$ oacillation. The probability that random fluctuations could produce a comparable signal has been found $8 \times 10^{-8}$, exceeding a $5 \sigma$ significance. The oscillation frequency $\Delta m_{s}$ bas been measured $17.77 \pm 0.10$ (stat.) \pm 0.07 (syst.) $\mathrm{ps}^{-1}$, (Fig. 2) which allows to extract $\left|V_{t d} / V_{t s}\right|=0.2060 \pm 0.0007$ (exp.) $+0.0081-0.0060$ (theor.). Fig. 2 reports also the signal mass distribution and the amplitude as a function of $\Delta m_{s}$ used for the recent D0 updated measurement $\left(\Delta m_{s}=18.53 \pm 0.93(\right.$ stat $) \pm 0.30($ syst $\left.)\right)[27]$.

\section{Conclusions}

In this paper we reviewed the techniques (detector elements and analysis techniques) used to perform heavy flavour physics analyses at CDF and D0. We described the CDF and D0 detectors and triggers and we provided some example analyses, involving the measurement of the $\mathrm{B}$ hadron masses, lifetimes, mixing and CP asymmetries. 


\section{References}

[1] D. Acosta et al., the CDF Collaboration, Phys. Rev. D 75, 012010 (2007).

[2] T. Aaltonen et al., the CDF Collaboration, Phys. Rev. D 79, 092003 (2009).

[3] A. Sill et al., Nucl. Instrum. Meth. A530:1 (2004).

[4] T. Affolder et al., Nucl. Instrum. Meth. A526:249 (2004).

[5] D. Acosta et al., Nucl. Instrum. Meth. A518:605 (2004).

[6] L. Balka et al., Nucl. Instrum. Meth. A267:272 (1988). M. Alborw. et al., Nucl. Instrum. Meth. A453:84 (2000).

[7] G. Ascoli et al., Nucl. Instrum. Meth. A268:33 (1988).

[8] E. J. Thomson et al., IEEE Trans. on Nucl. Sc. vol. 49, n. 3 (2002).

[9] A. Abulencia et al., IEEE Trans. Nucl. Sci. 55, 126-132 (2008).

[10] A. Bardi et al., Nucl. Instrum. Meth. A485:178 (2002).

[11] W. Ashmanskas et al., Nucl. Instrum. Meth. A518: 532, (2004)

[12] V. M. Abazov et al., Nucl. Instrum. Meth. Phys. res. A565, 463 (2006).

[13] V. M. Abazov et al., Phys. Rev. Lett. 101, 232002 (2008).

[14] T. Aaltonen et al., arXiv:0905.3123[hep-ex].

[15] V. M. Abazov et al., Phys. Rev. Lett. 94, 042001 (2005).

[16] V. M. Abazov et al., Phys. Rev. Lett. 94, 042001 (2005).

[17] , D. Abulencia et al., Phys. Rev. Lett. 98, 122001 (2007).

[18] , D. Acosta et al., Phys. Rev. Lett. 96, 202001 (2006).

[19] A. Abulencia et al., Phys. Rev. Lett. 98, 122001 (2006).

[20] http://www-cdf.fnal.gov/physics/new/bottom/080207.blessed-bs-lifetime/

[21] A. Abulencia et al., Phys. Rev. Lett 97, 211802 (2006).

[22] A. Aaltonen et al., Phys. Rev. Lett. 103, 031801 (2009).

[23] E. Baracchini and G. Isidori, Phys. Lett. B633 (2006) 309.

[24] T. Moulikk, for the D0 Collaboration, "Flavor tagging at Tevatron including calibration and control", arXiv:hep-ex/0701022v1.

[25] , T. Aaltonen et al., Phys. Rev. Lett. 97, 242003 (2006).

[26] V. M. Abazov et al., Phys. Rev. Lett. 97, 021802 (2006).

[27] D0 Collaboration, Conference-Note 5618-CONF. 

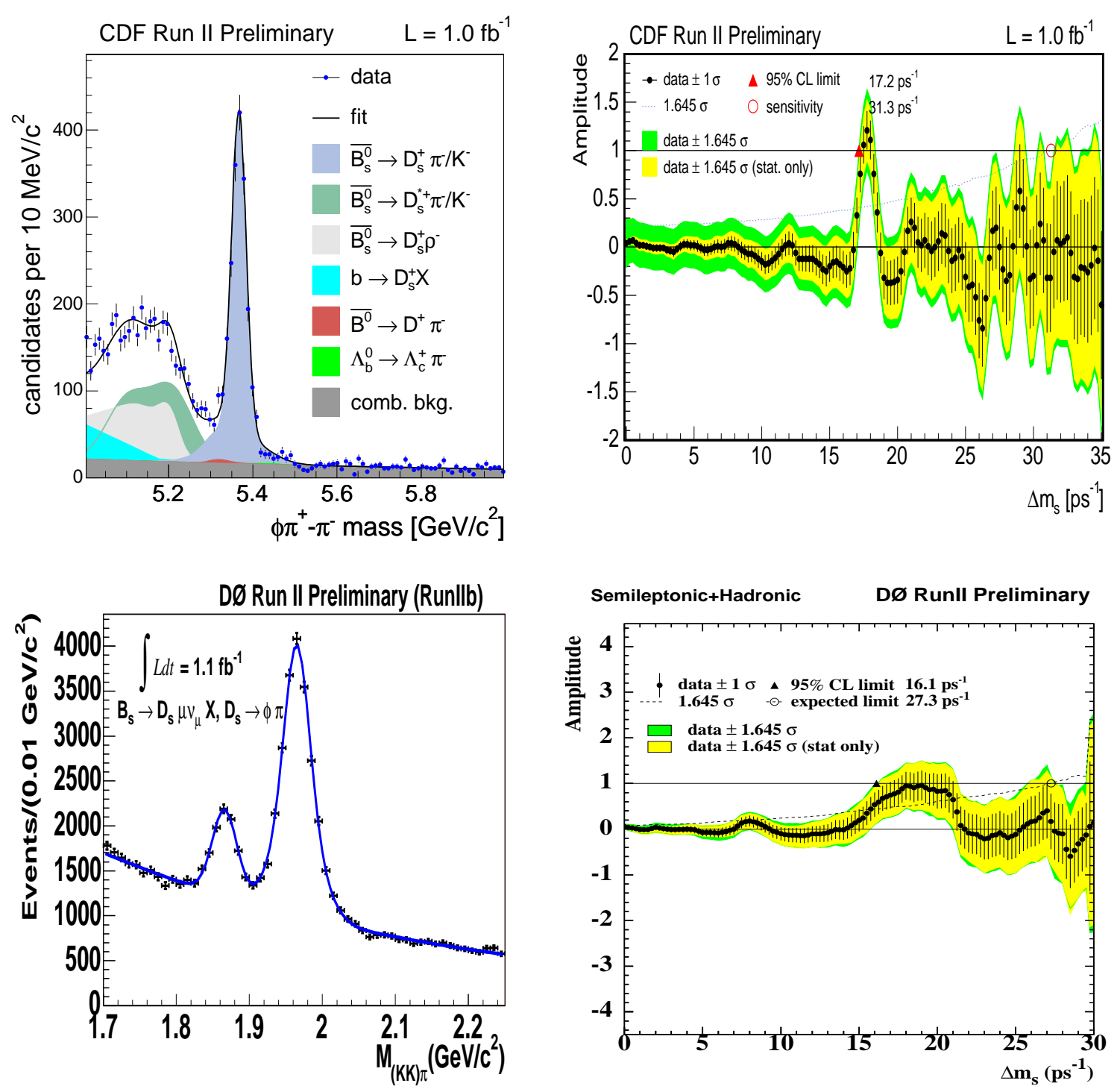

Figure 2: Top Left plot: Invariant mass distribution for $B_{s}^{0} \rightarrow D_{s}^{-}\left(\phi \pi^{-}\right) \pi^{+}$decays including some partially reconstructed decay modes contribution (drawn on top of the combinatiorial background); Top Right plot: Measured amplitude values and uncertainties versus $B_{s}^{0}-\bar{B}_{s}^{0}$ oscillation frequencey $\Delta m_{s}$.

Bottom Left plot: $D_{s}^{+}$invariant mass distribution in the $\mu \phi \pi$ decay mode for RunIIb data; the left and right peaks respectively correspond to the $\mu(e) D^{ \pm}$and $\mu(e) D_{s}$ candidates; Bottom Right plot: Combined (semileptonic+hadronic) amplitude as a function of $\Delta m_{s}$ with statisical and systematic uncertainties. 